\title{
A continuous GPS coordinate time series analysis strategy for high-accuracy vertical land movements
}

\author{
F. Norman Teferle ${ }^{\mathrm{a}, *}$, Simon D. P. Williams ${ }^{\mathrm{b}}$, Halfdan P. Kierulf ${ }^{\mathrm{c}}$, \\ Richard M. Bingley ${ }^{a}$, Hans-Peter Plag ${ }^{\mathrm{d}}$ \\ ${ }^{a}$ Institute of Engineering Surveying and Space Geodesy, University of Nottingham, \\ Nottingham NG7 2RD, UK \\ ${ }^{\mathrm{b}}$ Proudman Oceanographic Laboratory, Joseph Proudman Building, 6 Brownlow Street, \\ Liverpool L3 5DA, UK \\ ${ }^{\mathrm{c}}$ Norwegian Mapping Authority, Geodetic Institute, Kartverksveien 21, N-3511 Hønefoss, \\ Norway \\ ${ }^{\mathrm{d}}$ Nevada Bureau of Mines and Geology and Seismological Laboratory, University of \\ Nevada, Reno NV 89557, USA
}

\begin{abstract}
A CGPS coordinate time series analysis strategy was evaluated to determine highly accurate vertical station velocity estimates with realistic uncertainties. This strategy uses a combination of techniques to 1) obtain the most accurate parameter estimates of the station motion model, 2) infer the stochastic properties of the time series in order to compute more realistic error bounds for all parameter estimates, and 3) improve the understanding of apparent common systematic variations in the CGPS coordinate time series, which are believed to be of geophysical and/or technical origin. The strategy provided a pre-processing of the coordinate time series in which outliers and discontinuities were identified. Subsequent parameterization included a mean value, a constant rate, periodic terms with annual
\end{abstract}


and semi-annual frequencies, and offset magnitudes for identified discontinuities. All parameters plus the magnitudes of different stochastic noise were determined using Maximum Likelihood Estimation (MLE). Empirical Orthogonal Function (EOF) analysis was used to study both the temporal and spatial variability of the common modes determined by this technique. After outlining the CGPS coordinate time series analysis strategy this paper shows initial results for coordinate time series for a four year (2000-2003) period from a selection of CGPS stations in Europe that are part of the European Sea Level Service (ESEAS) CGPS network.

Key words: Global Positioning System, Coordinate Time Series, Maximum Likelihood Estimation, EOF Analysis, Common Mode

\section{Introduction}

Studies of changes in globally-averaged sea level based on tide gauge data and continuous GPS (CGPS) measurements require the determination of highly accurate vertical land movements with respect to a global, geocentric reference frame at the $1 \mathrm{~mm} / \mathrm{yr}$ level. Over the last decade, CGPS has evolved to be one of the few space geodetic techniques, which can potentially achieve such high accuracies in a globally homogeneous and practical manner. To this day, the challenge for CGPS remains in the consistent and accurate determination of the vertical coordinate component, as many error sources manifest themselves primarily in this coordinate. It was shown that the vertical coordinate component is largely affected by residual systematic effects due to inappropriately modelled tropospheric delay, antenna phase center variations or different loading processes (e.g. van Dam et al., 1994;

\footnotetext{
* Corresponding author

Email address: norman.teferle@nottingham.ac.uk (F. Norman Teferle).
} 
Mao et al., 1999; van Dam et al., 2001; Johansson et al., 2002; Boehm \& Schuh, 2004). Furthermore, biases and inconsistencies in the reference frame and satellite orbits, or CGPS processing strategy related effects, propagate into this coordinate component, increasing the noise level, introducing artificial long-term trends and/or common systematic variations. These variations often have a periodic nature and are correlated over large areas or continental regions (e.g. Wdowinski et al., 1997; Herring, 1999; Johansson et al., 2002; Kierulf et al., 2006).

In order to determine the secular change of the station position, i.e. the station velocity, with the required accuracy and realistic uncertainties, and to improve the understanding of the apparent, common systematic variations in CGPS coordinate time series, a complex time series analysis strategy is discussed and evaluated. After a pre-processing of the coordinate time series, this strategy incorporates Maximum Likelihood Estimation (MLE) and Empirical Orthogonal Function (EOF) analysis.

This study presents the initial evaluation of the coordinate time series analysis strategy. MLE and parts of the proposed EOF analysis have been applied to the coordinate time series obtained from six independent European Sea Level Service (ESEAS) CGPS Analysis Centres (AC). Using MLE, the best parameter estimates for the station motion model with realistic uncertainties are obtained. Furthermore, the stochastic properties of the coordinate time series also obtained from the MLE are discussed. Here a particular interest is the possibility of using the stochastic information to identify and/or separate the effects of different CGPS processing strategies, including strategy-implied reference frame definitions, the use of different orbit and clock products, and receiver antenna phase centre modelling, in the coordinate time series. The temporal and spatial variability of the coordinate time series is investigated using EOF analysis. The different modes, their geographical pattern and the associated common mode times series determined from the EOF analysis 
are discussed. It is believed that a better understanding of these modes describing the common, systematic variations in the coordinate time series will improve future parameterizations of the station motion model during CGPS processing and/or the analysis of coordinate time series. This improved modelling reduces the scatter in the residual coordinate time series, thus allowing further advances in the accurate determination of vertical station velocities and their uncertainties.

The following section describes previous work on the analysis of CGPS coordinate time series. In Section 3 the coordinate time series analysis strategy is introduced in more detail. This section also comprises a brief review of the station motion model and techniques applied. Section 4 then describes the results obtained for this initial evaluation of the coordinate time series analysis strategy.

\section{Previous Work}

Previous work on CGPS coordinate time series analysis has included much research on the effect of time-correlated (coloured) noise (e.g. Langbein \& Johnson, 1997; Zhang et al., 1997; Mao et al., 1999; Williams, 2003a; Langbein, 2004; Williams et al., 2004), the reduction of spatially correlated variations (e.g. Wdowinski et al., 1997; Nikolaidis, 2002; Johansson et al., 2002), annual and semi-annual harmonic signals (e.g. Blewitt \& Lavallée, 2002; Dong et al., 2002), and coordinate offsets (Williams, 2003b) on station velocity estimates and associated uncertainties. Furthermore, a monitoring and analysis strategy suggested for CGPS stations colocated with tide gauges, using pairs of CGPS stations, i.e. the dual-CGPS station concept, was discussed by Teferle et al. (2002a).

Although, it is now widely accepted that the assumption that daily variations in 
CGPS coordinate time series are purely random and time-independent is unrealistic, still only few research groups account for coloured noise in their analyses. Whereas random or white noise $(\mathrm{WN})$ can be greatly reduced by increasing the number of measurements and averaging, coloured noise is not or to a far lesser degree reduced by these measures. The general conclusion being that if coloured noise is not accounted for, station velocity uncertainties may be underestimated by up to an order of magnitude. It is, therefore, important to understand the stochastic properties of CGPS coordinate time series in order to obtain realistic uncertainties for all parameters estimated.

One way of describing noise is by means of a power-law process. It has been shown that many physical and geophysical processes approximate a process where the power spectrum has the form

$$
P_{x}(f)=P_{0}\left(f / f_{0}\right)^{\kappa}
$$

with $f$ being the spatial or temporal frequency, $P_{0}$ and $f_{0}$ being normalizing constants, and $\kappa$ being the spectral index (Agnew, 1992). For many phenomena the index $\kappa$ would fall in the range -3 to -1 with the integer cases $(\kappa=-1)$ and $(\kappa=-2)$ being flicker and random walk noise respectively. Classical white noise can be shown to have a spectral index $\kappa=0$. The term coloured (time-correlated) noise will be used to refer to power-law processes other than classical white noise.

By filtering the CGPS coordinate time series, common systematic variations, i.e. the spatial correlations in time series can be removed, enhancing local, stationspecific signals. The stacking method (Wdowinski et al., 1997; Nikolaidis, 2002; Wdowinski et al., 2004) based on computing a daily common mode improves the signal-to-noise ratio, which is especially important for the vertical component. Johansson et al. (2002) investigated the use of Empirical Orthogonal Function (EOF) 
analysis for this purpose. Using least-squares, they determined a combined set of parameters including velocity and offset parameters, admittance parameters for geophysical signals assumed to be correlated with the time series, and between-site correlation parameters. Then, they carried out an unweighted EOF analysis (Emery \& Thomson, 1989; Johnson \& Wichern, 1988) where eigenvectors and associated eigenvalues comprised different common modes with decreasing contributions to the total variance of the coordinate time series. This common mode construction differs to the stacking method as the weights of the spatial filter are obtained from the eigenvalues and not the standard errors of the daily position solutions.

\section{Methodology}

The methodology applied in this paper for CGPS coordinate time series analysis is based around the stochastic analysis using MLE and a more deterministic description of the data using EOF analysis. Prior to the application of these, the coordinate time series are cleaned for outliers (e.g. Wdowinski et al., 1997; Nikolaidis, 2002) and periods of bad data are identified and removed. The strategy also incorporates the detection of significant periodic signals, however, for this evaluation only annual and semi-annual harmonics are assumed. An important step in this pre-processing of the coordinate time series is also the detection and validation of coordinate offsets based on 1) a visual inspection of the raw time series, 2) International GNSS Service (IGS) site information log files, 3) earthquake files and

4) the use of a change-detection-algorithm (e.g. Williams, 2003b). Fig. 1 gives a schematic overview of the CGPS coordinate time series analysis strategy.

Figure 1 here 
The pre-processed coordinate time series are analysed using MLE to infer a set of highly accurate station velocity estimates with realistic uncertainties, fully accounting for coloured noise. This is the primary objective of the coordinate time series analysis strategy. However, in parallel, the strategy is also designed to allow investigations of the common, systematic variations apparent in many CGPS coordinate time series using EOF analysis. This improves the understanding, and supports the interpretation of these spatially correlated variations. The authors believe that the information gained from the combination of MLE and EOF analysis allows improvements in the parameterization of the station motion model, reducing the scatter in residual coordinate time series. This eventually leads to better station velocity estimates and uncertainties. In the following part of this section the station motion model, MLE and the EOF analysis are briefly described.

\subsection{Station Motion Model}

In the analysis of the coordinate time series, a model for the station movement is used, where the position of a point $x$ is given by

$$
x(t)=x_{0}+v_{0}\left(t-t_{0}\right)+\sum_{j=1}^{M} g_{j}(t, \vec{x})+\varepsilon(t)
$$

where $t$ is the time, $t_{0}$ the origin of time, $x_{0}$ the initial coordinate at time $t=t_{0}, v_{0}$ is a constant velocity of the point, $g_{j}$ are geophysical (or other) processes, which affect the point coordinates and $\varepsilon(t)$ is the error term. Some of the geophysical processes such as solid Earth tides and ocean tide loading are reasonably well modelled and have power mainly at diurnal and semi-diurnal frequencies such that they are included in the CGPS processing stage. The remaining geophysical processes such as earthquakes (co-, pre- and post-seismic), atmospheric and hydrological loading, human exploitation, long period transients and artificial events (such as non 
tectonic offsets) are applied at the time series analysis stage. However, for many of these processes the model uncertainty is large or unknown, or no model even exists. Therefore, they can either be estimated, e.g. earthquakes, offsets, periodic terms with annual or semi-annual frequencies, or absorbed into the error term, which is generally assumed to be noise unless otherwise shown.

\subsection{Maximum Likelihood Estimation}

The stochastic analysis of the coordinate time series is carried out using MLE (Langbein \& Johnson, 1997; Zhang et al., 1997; Mao et al., 1999; Williams, 2003a; Williams et al., 2004). The best fitting noise model is determined by maximizing the log-likelihood probability function:

$$
\ln [\operatorname{lik}(\hat{\mathbf{v}}, \mathbf{C})]=\frac{-1}{2}\left[\ln (\operatorname{det} \mathbf{C})+\hat{\mathbf{v}}^{T} \mathbf{C}^{-1} \hat{\mathbf{v}}+n \ln (2 \pi)\right]
$$

with respect to the post-fit residual vector, $\hat{\mathbf{v}}$, containing $n$ elements, and using a fully populated covariance matrix for observations,

$$
\mathbf{C}=a^{2} \mathbf{I}+b_{\kappa}^{2} \mathbf{J}_{\kappa}
$$

described by a combination of white and power-law noise with amplitudes $a$ and $b_{\kappa}$ respectively. The identity matrix $\mathbf{I}$ is the covariance matrix of the white noise, resembling the time-independence of this noise process. $\mathbf{J}_{\kappa}$, the covariance matrix of the power-law noise is computed by means of fractional differencing (Hosking, 1981; Williams, 2003a) such that

$$
\mathbf{J}_{\kappa}=\mathbf{T} \mathbf{T}^{T}
$$


where $\mathbf{T}$ is a transformation matrix obtained from

$$
\mathbf{T}=\Delta T^{-\kappa / 4}\left[\begin{array}{ccccc}
\psi_{0} & 0 & 0 & \ldots & 0 \\
\psi_{1} & \psi_{0} & 0 & \ldots & 0 \\
\psi_{2} & \psi_{1} & \psi_{0} & \ldots & 0 \\
\vdots & \vdots & \vdots & \ddots & 0 \\
\psi_{n-1} & \psi_{n-2} & \psi_{n-3} & \ldots & \psi_{0}
\end{array}\right]
$$

with sampling interval $\Delta T$ and with

$$
\psi_{n}=\frac{-\frac{\kappa}{2}\left(1-\frac{\kappa}{2}\right) \ldots\left(n-1-\frac{\kappa}{2}\right)}{n !}=\frac{\Gamma\left(n-\frac{\kappa}{2}\right)}{n ! \Gamma\left(-\frac{\kappa}{2}\right)}
$$

As $n \rightarrow \infty, \psi_{n} \sim n^{-\frac{\kappa}{2}-1} / \Gamma\left(-\frac{\kappa}{2}\right)$ (Williams, 2003a).

Using MLE, precise stochastic models can be fitted to the coordinate time series, estimating the noise amplitudes for a model assuming a combination of white and power-law noise (WN+PLN). This recent approach is based on a general form of the power-law co-variance matrix, allowing the noise amplitudes and the spectral index to be estimated concurrently with all other parameters of the station motion model. The stochastic properties are estimated alongside the linear parameters in an iterative sense via a maximizing function. The maximizing function chooses a noise model and estimates the linear parameters upon which a new set of residuals is computed. Using these residuals and the co-variance matrix the log-likelihood value is estimated and a new noise model is chosen with, hopefully, a greater loglikelihood. This process is repeated until the log-likelihood probability function has been maximized. 
By pre-selection of a specific noise model for the maximization it is possible to investigate the fit of different stochastic models to the residual time series, e.g. the combination of white plus an integer case of power-law noise (Langbein \& Johnson, 1997; Zhang et al., 1997; Mao et al., 1999; Williams et al., 2004). Here, no preselection takes place, i.e. the a-priori noise model is not constrained to a particular case, therefore the MLE (WN+PLN) gives the most likely stochastic description of the coordinate time series (based on the initial assumption on the form of coloured noise), with the best station motion model parameter estimates (in a least-squares sense) and more realistic uncertainties.

\subsection{EOF Analysis}

EOF analysis (Lorenz, 1956; Emery \& Thomson, 1989; Johnson \& Wichern, 1988; Björnsson \& Venegas, 1997) is a decomposition of the data set in terms of orthogonal basis functions determined from the data themselves. It provides a compact description of the spatial and temporal variability of time series in terms of these orthogonal functions (EOFs) or statistical modes. The aim is to identify those modes that contribute the most variance to the coordinate time series of a CGPS network, exhibiting spatial and temporal correlation, i.e. common systematic variations. The first mode contains most of these variations. The second mode represents the function orthogonal to the first mode, representing most of the remaining variance, etc. It can be shown that, often, the first few modes are linked to other factors of technical or geophysical origin. For instance, in CGPS coordinate time series, spatial correlations due to snow accumulation on GPS antennas or radomes, for those antennas with radomes, are to a degree absorbed by these few modes (Johansson et al., 2002). In the following, the main elements of the theory are summarized according 
to Emery \& Thomson (1989); Johnson \& Wichern (1988); Björnsson \& Venegas (1997).

Let $x_{i j}$ be zero mean time series of measurements each with $i=1, \ldots, n$ data samples at $j=1, \ldots, p$ spatially distributed locations. Then, a data matrix $\boldsymbol{X}$ can be formed, containing the observation time series as column vectors and a map of the measurements at time $i$ as row vectors such that

$$
\underset{(n \cdot p)}{\mathbf{X}}=\left[\begin{array}{cccc}
x_{11} & x_{12} & \ldots & x_{1 p} \\
x_{21} & x_{22} & \ldots & x_{2 p} \\
\vdots & \vdots & & \vdots \\
& & & \\
x_{n 1} & x_{n 2} & \ldots & x_{n p}
\end{array}\right]
$$

The symmetric co-variance matrix $\boldsymbol{\Sigma}$ of $\boldsymbol{X}$ is defined by (Emery \& Thomson, 1989):

$$
\underset{(p \cdot p)}{\boldsymbol{\Sigma}}=\frac{1}{n-1} \underset{(p \cdot n)(n \cdot p)}{\boldsymbol{X}} \underset{\boldsymbol{X}}{\boldsymbol{X}}
$$

The eigenvalue problem to be solved is then:

$$
\underset{(p \cdot p)(p \cdot p)}{\boldsymbol{E}}=\underset{(p \cdot p)(p \cdot p)}{\boldsymbol{E}}
$$

$\Lambda$ is a square diagonal matrix containing the eigenvalues $\lambda_{j}$ sorted in descending order. $\boldsymbol{E}$ is a square orthogonal matrix with columns of normalized eigenvectors $\boldsymbol{e}_{\boldsymbol{j}}$ corresponding to the eigenvalues in $\Lambda$. Since $\Sigma$ is a symmetric, quadratic positive definite matrix, $\boldsymbol{E}$ has the property that $\boldsymbol{E}^{-1}=\boldsymbol{E}^{T}$ and so Eq. 10 can be written as

$$
\underset{(p \cdot p)}{\boldsymbol{\Sigma}}=\underset{(p \cdot p)(p \cdot p)(p \cdot p)}{\boldsymbol{E}} \underset{j=1}{\boldsymbol{E}^{\boldsymbol{T}}}=\sum_{j=1}^{p} \lambda_{j} \boldsymbol{e}_{\boldsymbol{j}} \boldsymbol{e}_{\boldsymbol{j}}^{\boldsymbol{T}}
$$

In $\boldsymbol{E}$, the $j$-th mode is simply the $j$-th eigenvector $\boldsymbol{e}_{\boldsymbol{j}}$ of $\boldsymbol{\Sigma}$ and the corresponding eigenvalue $\lambda_{j}$ gives a measure of the explained variance by the $j$-th mode. The 
contribution of the $j$-th mode to the total variance, also denoted as loading, is then explained in percentage as:

$$
\frac{100 \lambda_{j}}{\sum_{j=1}^{p} \lambda_{j}} \%
$$

The accumulated loading is the fraction of the total variance in $\%$ explained by the first $j$ modes.

The eigenvector matrix $\boldsymbol{E}$ has the property that $\boldsymbol{E}^{T} \boldsymbol{E}=\boldsymbol{E} \boldsymbol{E}^{T}=\boldsymbol{I}$ (where $\boldsymbol{I}$ is the identity matrix), which means that the modes are uncorrelated over space, i.e. they are orthogonal to each other, hence the name EOF. Plotting the different modes as a map gives the pattern of a standing oscillation. The time evolution of the mode, i.e. the amplitude time series or common mode time series, shows how this pattern oscillates in time. The time evolution of each mode contained in the columns of matrix $\boldsymbol{Y}$ can be obtained by a projection of the data matrix $\boldsymbol{X}$ onto the eigenvector matrix $\boldsymbol{E}$ such that

$$
\underset{(n \cdot p)}{\boldsymbol{Y}}=\underset{(n \cdot p)(p \cdot p)}{\boldsymbol{X}}
$$

Therefore, for the $j$-th mode the corresponding time evolution is the $j$-th column vector in $\boldsymbol{Y}$, and just as the modes are uncorrelated in space, the common mode time series are uncorrelated in time.

Since $\boldsymbol{E}$ is orthogonal, a complete reconstruction of the original data matrix is achieved from the matrix of the common mode time series and the eigenvector matrix and is given by

$$
\underset{(n \cdot p)}{\boldsymbol{X}}=\underset{(n \cdot p)(p \cdot p)}{\boldsymbol{Y}}
$$

Since the eigenvectors in $\boldsymbol{E}$ are orthogonal, Eq. 13 and Eq. 14 also hold for any $q<p$. These representations, i.e. truncated representations $\boldsymbol{X}^{(q)}$ of the data, use only the first $q$ modes with relatively large eigenvalues to extract the common systematic variations from a set of time series. Values of $q$ of up to 5 result in trun- 
cated representations capturing most of the total variance of the time series. The contributions from the remaining modes are generally assumed to be random noise (Björnsson \& Venegas, 1997).

\section{Results}

In this initial strategy evaluation only the vertical coordinate component is discussed. The results presented have been obtained for nine different CGPS stations: Aberdeen (ABER), Alacante (ALAC), Borkum (BORK), Cascais (CASC), Genoa (GENO), Helgoland (HELG), Liverpool (LIVE), Lowestoft (LOWE) and Newlyn (NEWL), which are part of the ESEAS CGPS network (Fig. 2). The data for all nine stations has been processed by six different ACs:

- General Command of Mapping (GCM), Ankara, Turkey.

- Norwegian Mapping Authority (NMA), Hønefoss, Norway.

- Royal Naval Observatory of Spain (ROA), Cadiz, Spain.

- Space Research Centre, Polish Academy of Science (SRC), Warszawa, Poland.

- Institute of Engineering Surveying and Space Geodesy, University of Nottingham (UNOTT), Nottingham, United Kingdom.

- Universidad Politécnica de Cataluña (UPC), Barcelona, Spain.

The main differences in the CGPS analyses between the ACs are the use of different scientific GPS softwares, CGPS processing strategies, satellite orbit and clock products and receiver antenna phase centre models (Tab. 1). The exact details of these analyses can be found in Kierulf et al. (2006). Since the results from SRC and UNOTT have been computed, an error was found in the Bernese GPS software, which was described to introduce annual variations ( $\sim 1 \mathrm{~cm}$ amplitude) in the 
vertical coordinate component of mid-latitude stations in large regional and global networks (Fridez, 2004). Although, re-computations at both SRC and UNOTT are underway, these corrected results were not available to the authors at the time of writing.

For the coordinate time series from UNOTT it was possible to use the stacking method (Wdowinski et al., 1997; Nikolaidis, 2002) to largely reduce the effect of this software error, which was assumed to be nearly constant over the extent of the CGPS network. As this error affected many users in the scientific GPS community it was deemed as important to include the results from SRC and UNOTT in this paper where possible.

Table 1 here

Figure 2 here

\subsection{MLE}

The height time series for six CGPS stations ALAC, CASC, GENO, HELG, LOWE and NEWL, considered to be of high quality, from the different ACs have been analysed using MLE (WN+PLN). As an example, Fig. 3 shows the height time series for ALAC for all ACs, for the period 2000 to 2003 inclusive. For UNOTT, both the unfiltered (UNOTT_unflt) and filtered (UNOTT_flt) height time series are shown. The filtered height time series have been obtained using the stacking method, which was based on the coordinate time series of 14 CGPS stations: ALAC, ANDO, BORK, CAMB, CASC, CEUT, GENO, HELG, LAGO, LIVE, NEWL, PMTG, SHEE and TGDE (Fig. 2). These 14 stations were selected on the basis that their coordinate time series are of high quality with no or few offsets and that data were available 
for most of the timespan used in this study. Furthermore, stations with site-specific problems, i.e. multipath and/or radio frequency interference are excluded (Teferle et al., 2003). This selection process reduces the chance that the daily common mode is affected by errors related to stations used in its computation. Similar approaches have previously been applied in Nikolaidis (2002); Teferle et al. (2002b); Wdowinski et al. (2004).

Clearly visible are the different systematic variations in the height time series for ALAC from the different ACs, which have been attributed mainly to the different CGPS processing strategies, softwares, orbit and clock products, and the receiver antenna phase centre modelling (Kierulf et al., 2006). The best agreement in the height time series is obtained for the solutions from NMA and ROA, which are both PPP solutions from GIPSY OASIS II together with JPL orbit and clock products (Tab. 1). Interestingly, the effect of the different modelling of the receiver antenna phase centres in these solutions does not seem to be visible in the height time series as both show very similar variations. The variations of the height time series of the third PPP solution computed by UPC differ from those of ROA and NMA. The most likely cause for this is the omission of tropospheric gradients in the modelling of the atmosphere by UPC, which has been reported by Kierulf et al. (2006) to affect the temporal characteristics of the time series.

The height time series for ALAC from GCM, NMA, ROA and UPC all show annual periodic variations with amplitudes between 1 to $2.3 \mathrm{~mm}$ and considerable variance in their phases. The SRC and UNOTT_unflt height time series, however, are dominated by a large $(\sim 1 \mathrm{~cm}$ amplitude) periodic signal with annual frequency. For UNOTT_unflt this signal is believed to be mainly a consequence of the Bernese 4.2 software error. However, for SRC this signal is thought to be a mixture of the same software error and the reference frame implied by the CGPS processing strategy 
used by SRC (Kierulf et al., 2006).

Figure 3 here

The vertical station velocity estimates of the six CGPS stations ALAC, CASC, GENO, HELG, LOWE and NEWL from all six ACs agree at varying levels to within approximately $\pm 3 \mathrm{~mm} / \mathrm{yr}$ (Fig. 4). Clearly, this level of disagreement can be attributed to differences in the height time series for a particular station obtained by the individual ACs, as in the example for ALAC from Fig. 3. The best agreement in the velocity estimates was obtained for the PPP solutions from NMA and ROA. The differing velocity estimates of the third PPP solution computed by UPC are assumed to be due to a combination of 1) the use of a different cut-off elevation angle (Kierulf et al., 2006) and 2) the UPC's shorter height time series.

Comparing the velocity uncertainties from the MLE (WN+PLN) with those from MLE with a white noise only model, it was confirmed that assuming white noise only would lead to an underestimation of the velocity uncertainties. It can be shown that for GCM and UNOTT the error bounds would be too optimistic by a factor of on average of 4, whereas for NMA, ROA, SRC and UPC by a factor of on average of 3. These findings confirm those of previous analyses and highlight the fact that the stochastic analysis of the CGPS coordinate time series is necessary in order to obtain realistic uncertainties.

\section{Figure 4 here}

The parameters for annual and semi-annual harmonic signals and height offsets, were also obtained from the MLE (WN+PLN). However, these also vary considerably due to the differences in the height time series, as discussed in Kierulf et al. (2006), and are not discussed any further here. 
The stochastic properties of the height time series are described using the spectral index $\kappa$ and the noise amplitudes for white and power-law noise, $a$ and $b_{\kappa}$, respectively. Fig. 5 shows the spectral indices for the six CGPS stations ALAC, CASC, GENO, HELG, LOWE and NEWL for each AC. It can be seen that all estimated indices are in the range from -1.2 to -0.4 , i.e. stretching from fractional Brownian to fractional Gaussian (white) noise, including flicker noise at -1.0 (Agnew, 1992). The station-specific mean spectral indices and their standard deviations about the mean showed the best agreement between the different ACs for ALAC and LOWE, and the worst for GENO. GENO was also identified to have the lowest stationspecific mean spectral index $(-0.8 \pm 0.2)$. The AC-specific mean spectral indices and standard deviations about the mean suggested that the most consistent spectral indices were obtained for the RN-DD solutions from GCM and SRC. Furthermore, the GPS processing strategy-specific mean spectral indices and standard deviations computed for PPP (NMA, ROA, UPC) and RN-DD (GCM only and GCM, SRC) solutions were nearly equivalent with $-0.7 \pm 0.2,-0.7 \pm 0.1$ and $-0.6 \pm 0.1$, respectively. Figure 5 also seems to suggest that the use of the different receiver antenna phase centre modelling by NMA does not affect the stochastic properties of the height time series.

\section{Figure 5 here}

Tab. 2 shows the analysis-centre-specific, the station-specific and the CGPS processing strategy-specific mean noise amplitudes computed for the individual white and power-law noise amplitudes obtained from the MLE (WN+PLN). When considering the analysis-centre-specific mean noise amplitudes, $a_{m}$ is estimated to be between 3.1 and $5.3 \mathrm{~mm}$, whereas $b_{\kappa m}$ is 3 to 4 times larger and between 10.9 and $20.4 \mathrm{~mm} \mathrm{yr}^{\kappa / 4}$. Although the mean noise amplitudes for NMA and ROA are nearly equal, their standard deviations vary with those for NMA being nearly double the 
size than those for ROA. Considering that the only difference between these PPP solutions is the modelling of the receiver antenna phase centres, it must be assumed that the increase in variability of the mean noise amplitudes for NMA is an effect of this subtle modelling difference.

Clearly visible from Tab. 2 is the fact that the mean power-law noise amplitudes for SRC and UNOTT are significantly larger, and as indicated by their standard deviations more varied, than for the other ACs. This is assumed to be a direct consequence of the Bernese 4.2 software error. By comparing the mean power-law noise amplitudes from UNOTT_unflt and UNOTT_flt, the stacking method demonstrated its capability to reduce noise levels at all frequencies in the presence of spatially correlated variations. These results also indicate that only some portion of the power of the common mode is at annual and semi-annual frequencies.

Due to the obvious effect of the software error on the analysis-centre-specific mean power-law noise amplitudes for SRC and UNOTT, the individual noise amplitudes from SRC and UNOTT were excluded from the computation of station-specific and CGPS processing-strategy-specific mean noise amplitudes in Tab. 2.

Looking at the station-specific mean noise amplitudes it can be argued that for stations ALAC, CASC, GENO and LOWE, $a_{m}$ and $b_{\kappa m}$ are of similar magnitude when compared to the mean noise amplitudes for HELG and NEWL. For HELG, $a_{m}$ is close to 0 . This is due to the fact that the spectral indices for HELG from GCM, NMA, ROA and UPC are on average small (Fig. 5). In such cases, the powerlaw noise amplitude includes most or all of the white noise amplitude as the total noise content can now be explained by power-law noise only, which is close to classical white noise. NEWL shows less power for white noise than ALAC, CASC, GENO and LOWE, but more power for coloured noise than the other stations. In 
general, some portion of an increase in coloured noise can be explained by the spectral index being lower.

The CGPS processing strategy-specific mean noise amplitudes $a_{m}$ and $b_{\kappa m}$ have been computed for two different strategies: PPP using the solutions from NMA, ROA and UPC, and RN-DD based on the solutions from GCM. At this stage, this comparison does not allow to identify a strategy-related induced noise component. Therefore, based on the data set analysed, it is currently not possible to differentiate between CGPS processing strategies using strategy-specific mean noise amplitudes.

Table 2 here

\subsection{EOF analysis}

Within this coordinate time series analysis strategy the EOF analysis is the method of choice for improving the understanding of the common systematic variations observed in CGPS coordinate time series. In order to achieve this, the strategy involves the stochastic analysis of the common mode time series obtained from the EOF analysis (Fig. 1). However, due to the large differences in the height time series from the ACs (e.g. see Fig. 3) and their implications on the currently ongoing development of the CGPS processing strategies (Kierulf et al., 2006), the EOF analysis and the subsequent MLE have, at this stage, not been carried out as outlined in Fig. 1. In this initial study only the contributions of the different modes to the total variance of the time series, the spatial pattern of the first three modes and the time evolution of the first three modes, are presented.

The results of the EOF analysis are based on the height time series for nine stations 
of the ESEAS CGPS network: ABER, ALAC, BORK, CAMB, CASC, HELG, LIVE, LOWE and NEWL. These stations were considered as good stations which showed few data gaps, had at least 2.5 years of data and had a weighted RMS of less than $7 \mathrm{~mm}$ for the height time series from NMA. Furthermore, the analysis was carried out separately for the time series from each of the five ACs and used an unweighted EOF analysis (Emery \& Thomson, 1989; Johnson \& Wichern, 1988).

Tab. 3 shows the accumulated loadings from the EOF analysis for the time series results from five different ACs. For the height time series from NMA, ROA and SRC the 1 st mode represents approximately $45 \%$ of the total loading, which represents to a large degree the common systematic variations in the height time series for the nine stations. It should be noted that both NMA and ROA use PPP (Tab. 1). The UNOTT flt solution is also based on PPP, however, as the common mode based on the stacking method has already been removed, the contribution of the 1 st mode to the total variance represents only as little as $28 \%$.

\section{Table 3 here}

The comparison of the common mode time series and the spatial pattern of the modes has at this stage only been carried out for results from NMA and GCM, representing both PPP and RN-DD solutions, respectively. According to Tab. 3 the accumulated loadings of the first three modes for the results from NMA and GCM explain 66 and $70 \%$ of the total variance, respectively. The associated time series for these modes (Fig. 6) show very different variations for NMA and GCM. It can be noticed that for the PPP solution from NMA the common mode time series show annual and semi-annual signals with nearly equal amplitudes, whereas for the RNDD solution from GCM the annual harmonic dominates in all three modes.

The first three modes for NMA and GCM show very regular spatial patterns (Fig. 7). 
The pattern of the 1st mode shows a positive loading for all stations. However, where the PPP solution from NMA shows almost the same loading for all stations, the RN-DD solution from GCM shows an east-west gradient with minimal loading coefficients for stations in central Europe. For the RN-DD solutions, the pattern of 2 nd mode has a north south gradient, while the pattern of the 3 rd mode has no clear geographic variations. For the PPP solution the pattern of the 2nd and 3rd modes show a clear geographical pattern oriented around the northwest-southeast and southwest-northeast axis, respectively.

Figure 6 here

Figure 7 here

\section{Discussion}

The height time series from NMA, ROA, and UPC are obtained using PPP solutions and, hence, display all geocentric station motion, i.e. all motions with respect to the origin of the reference frame, not modelled in the station motion model. The height time series from GCM are obtained using RN-DD solutions, realizing a CGPS processing strategy-implied regional reference frame by constraining the coordinates of nine CGPS station in Europe to their ITRF2000 values (Kierulf et al., 2006).

For analyses comparable to those of NMA, ROA and UPC, Williams et al. (2004) found that these are best described by a combination of white and power-law noise with a mean spectral index of $-0.8 \pm 0.4$. This is in excellent agreement with the CGPS processing strategy-specific mean spectral index of $-0.7 \pm 0.2$ obtained for NMA, ROA and UPC in this study. For regionally filtered solutions, Williams et al. (2004) obtained a slightly lower mean spectral index (-0.9), which was shown to be 
independent of the CGPS processing strategy. Although, the spectral indices were computed for unfiltered height time series, the value of -0.8 for the AC-specific mean spectral index for GCM is close to that found by Williams et al. (2004).

Unfortunately, it is not straight forward to compare the white and power-law noise amplitudes estimated by the MLE (WN+PLN) of this study to previously published values. Williams et al. (2004) only showed amplitudes for white and flicker noise, a special integer case of power-law noise, which are not directly comparable. However, they detected differences in the stochastic properties of the global solutions based on the double difference and PPP approaches. This is encouraging with respect to future investigations by the authors once the complete ESEAS CGPS network has been analysed.

Johansson et al. (2002) investigated the use of EOF analysis to describe and remove common systematic variations in the coordinate time series of a regional CGPS network and produced a set of station velocity estimates based on regionally filtered coordinate time series. Their RMS differences in the vertical station velocity estimates of the solution based on the EOF analysis with respect to their standard solution was at the $1.4 \mathrm{~mm} / \mathrm{yr}$ level. Also Wdowinski et al. (2004) and Kierulf et al. (2006) concluded that the station velocity estimates obtained from regionally filtered coordinate time series are shifted and decoupled from the reference frame introduced in the GPS analysis. Within ESEAS, the estimated vertical station velocities are required to be with respect to a global, geocentric reference frame. Therefore, velocity estimates for filtered coordinate time series, based on either the stacking method or the EOF analysis, cannot be used.

However, the authors argue that it is possible to obtain additional information about the common systematic variations observed in time series from both these tech- 
niques. Williams et al. (2004) investigated the stochastic properties of the common mode removed from regional CGPS network analyses and concluded that the common mode is most probably a combination of white and flicker noise. The results from the EOF analyses of the ESEAS CGPS network obtained so far look promising in that, in combination with MLE, it may be possible to attribute different CGPS processing strategy-specific stochastic properties to the common mode time series and, hence, obtain a better understanding of the spatially correlated variations observed in CGPS coordinate time series.

\section{Conclusions}

A CGPS coordinate time series analysis strategy for the determination of highly accurate vertical station velocity estimates and realistic uncertainties has been introduced and evaluated. As outlined, this strategy incorporates a pre-processing of the coordinate time series and subsequently determines the best parameter estimates for the station motion model together with the stochastic properties of the time series. Using the stochastic information, realistic uncertainties for the estimated parameters can be obtained. Furthermore, using EOF analysis, the strategy allows a decomposition of the coordinate time series into different modes and common mode time series, which can be used to investigate apparent common systematic variations in the coordinate time series.

Preliminary height time series, for the period from 2000 to 2003, for six CGPS stations from six ESEAS CGPS analysis centres have been analysed using MLE. The height time series show varying agreement, with some exhibiting large systematic effects partly imposed by the CGPS processing strategy. 
The stochastic properties of the CGPS coordinate time series confirmed the presence of coloured noise. The mean spectral indices are consistent for all analysis centres, with slightly smaller standard deviations for GPS network solutions applying the double difference approach. The analysis-centre-specific mean noise amplitudes show small differences, which may be CGPS processing strategy related. However, the CGPS processing strategy-related mean noise amplitudes can currently not differentiate between solutions using the precise point positioning or double difference approach.

The EOF analysis was applied to the preliminary height time series, for the period from 2000 to 2003, for nine CGPS stations from five ESEAS CGPS analysis centres. This initial study showed that up to $75 \%$ of the total variance is contained in the first three modes. Furthermore, the EOF analysis showed that the characteristics of the common mode time series are affected by the CGPS processing strategy.

The results of this initial evaluation of the CGPS coordinate time series analysis strategy are encouraging, in highlighting the potential of using both MLE and EOF analysis in combination to determine highly accurate vertical station velocity estimates with realistic uncertainties, and to improve the understanding of apparent common systematic variations in coordinate time series. However, they also reflect the large differences found in the current height time series, which require more investigations into the CGPS processing strategies.

\section{Acknowledgements}

This work was carried out under the European Sea Level Service Research Infrastructure (ESEAS-RI) project and is funded by the European Commission Frame- 
work 5 Contract No. EVR1-CT-2002-40025. The authors would like to thank the IGS, JPL, the European Reference Frame (EUREF), the Norwegian Mapping Authority, the British Isles GPS archiving Facility (http://www.bigf.ac.uk) and ESEAS for the provision of CGPS data and products. Furthermore, thanks go to Trond Haakonsen, Etienne Orliac and Cristina Garcia Silva for their contributions.

\section{References}

Agnew, D.C. (1992), The Time Domain Behavior of Power-Law Noises, Geophys. Res. Letts., 19, 333-336.

Björnsson, H., Venegas, S. A. (1997), A Manual of EOF and SVD Analysis of Climatic Data, Dept. of Atmos. \& Ocean. Sci. and Cent. for Clim. \& Glob. Change Res., McGill University, pp. 52.

Blewitt, G. \& Lavallée, D. (2002), Effect of Annual Signals on Geodetic Velocity, J. Geophys. Res., 107 (B7), 10.1029/2001JB000570.

Boehm, J. \& Schuh, H. (2004), Vienna mapping functions in VLBI, Geophys. Res. Lett., 31, L01603, 10.1029/2003GL018984.

Dong, D., Fang, P., Bock, Y., Cheng, M. K. \& Miyazaki, S. (2002) Anatomy of apparent seasonal variations from GPS derived site position time series, J. Geophys. Res., 107(B4), doi:10.1029/2001JB000573.

Emery, J. E. and R. E. Thomson (1989), Data Analysis in Physical Oceanography, Pergamon.

Fridez, P. (2004) Error number_9 [online]. Berne: The Astronomical Institute University of Berne. Available at $<$ http://www.bernese.unibe.ch/ support.html> [Accessed 25 February 2005].

Herring, T. A. (1999), Geodetic Applications of GPS, Proc. IEEE, 87 (1), 92-110. 
Herring, T. A. (2003), GLOBK: Global Kalman filter VLBI and GPS Analysis program version 5.08, Massachusetts Institute of Technology (MIT). Cambridge.

Hosking, J. R. M. (1981), Fractional Differencing, Biometrika, 68 (1), 165-176.

Hugentobler, U., Schaer, S. Fridez, P. (2001), Bernese GPS Software Version 4.2, University of Berne, February 2001.

Johansson, J. M., Davies, J. L., Scherneck, H. G., Milne, G. A., Vermeer, M., Mitrovica, J. X., Bennett, R. A., Jonsson, B., Elgered, G., Elosegui, P., Koivula, H., Poutanen, M., Rønnaeng, B. O., \& Shapiro, I. I. (2002) Continuous GPS measurements of postglacial adjustment in Fennoscandia 1. Geodetic Results, J. Geophys. Res., 107 (B8), ETG 3/1-3/27.

Johnson, R. A. and D. W. Wichern (1988), Applied Multivariate Statistical Analysis, Prentice-Hall.

Johnson, H. O., Wyatt, F. (1994), Geodetic network design for fault-mechanics studies, Manuscripta Geodetica, 19, 309-323.

Kierulf, H.P., Plag, H.-P. (2004), Reference Frame induced noise in CGPS coordinate time series, EOS Trans., AGU,85(47), Fall Meet. Suppl., Abstract G53A0114 .

Kierulf, H. P., Plag, H.-P., Bingley, R. M., Teferle, F. N., Demir, C., Cingoz, A., Yildiz, H., Garate, J., Davila, J. M., Garcia-Silva, C., Zdunek, R., Jaworski, L., Martinez-Benjamin, J. J., Orus, R., Aragon, A. (2006), Comparison of GPS analysis strategies for high-accuracy vertical land motion, Phys. Chem. Earth, this issue.

King, R. W., Bock, Y. (2003), Documentation for the GAMIT analysis software, release 10.1, Massachusetts Institute of Technology (MIT), Cambridge.

Langbein, J. (2004), Noise in two-color electronic distance meter measurements revisited, J. Geophys. Res., 109, B04406, 10.1029/2003JB002819.

Langbein, J., Johnson, H. O. (1997), Correlated errors in geodetic time series: Im- 
plications for time-dependent deformation, J. Geophys. Res., 102, 591-603.

Lorenz, E. N. (1956), Empirical orthogonal functions and statistical weather prediction, Sci. rep. no. 1, statistical forecasting project, M.I.T., Cambridge, MA, USA, 48 pp.

Mao, A., C. Harrison, and T. H. Dixon (1999), Noise in GPS Coordinate Time Series, J. Geophys. Res., 104, 2797-2818.

Nikolaidis, R. M. (2002), Observation of geodetic and seismic deformation with the global positioning system, $\mathrm{PhD}$ thesis, University of California.

Teferle, F. N., Bingley, R. M., Dodson, A. H., \& Baker, T. F. (2002a) Application of the dual-CGPS concept to monitoring vertical land movements at tide gauges, Phys. Chem. Earth, 27 (32-34), 1401-1406.

Teferle, F. N., Bingley, R.M., Dodson, A. H., Penna, N. T., \& Baker T.F. (2002b) Using GPS to separate crustal movements and sea level changes at tide gauges in the UK, H. Drewes, A. H. Dodson, L. P. S. Fortes, L. Sanchez, \& P. Sandoval, eds., in: Vertical Reference Systems, Springer-Verlag, Heidelberg Berlin, pp. 264269.

Teferle, F. N., Bingley, R. M., Dodson, A. H., Apostoloidis, P. \& Staton, G. (2003) RF Interference and Multipath Effects at Continuous GPS Installations for Longterm Monitoring of Tide Gauges in UK Harbours, in Proc. 16th Tech. Meeting of the Satellite Division of the Inst. of Navigation, ION GPS/GNSS 2003, Portland, Oregon, 9-12 September 2003, pp. 12.

van Dam, T. M., Blewitt, G., \& Heflin, M. B. (1994) Atmospheric pressure loading effects on Global Positioning System coordinate determinations, J. Geophys. Res., 99 (B12), 23,939-23,950.

van Dam, T. M., Wahr, J., Milly, P. C. D., Shmakin, A.B., Blewitt, G., Lavallée, D., \& Larson, K. M. (2001) Crustal Displacements due to continental water loading, Geophys. Res. Lett., $28(4), 651-654$. 
Williams, S. D. P. (2003a), The effect of coloured noise on the uncertainties of rates estimated from geodetic time series, J. Geodesy, 76, 483-494.

Williams, S. D. P. (2003b), Offsets in Global Positioning System Time Series, J. Geophys. Res., 108 (B6), 2310, 10.1029/2002JB002156.

Williams., S. D. P., Y. Bock, P. Fang, P. Jamason, R. M. Nikolaidis, L. Prawirodirdjo, M. Miller, and D. J. Johnson (2004), Error analysis of continuous GPS position time series, J. Geophys. Res., 109, B03412, 10.1029/2003JB002741.

Wdowinski, S., Y. Bock, J. Zhang, P. Fang, and J. Genrich (1997), Southern California Permanent GPS Geodetic Array: Spatial Filtering of Daily Positions for Estimating Coseismic and Postseismic Displacements induced by the 1992 Landers Earthquake, J. Geophys. Res., 102 (B8), 18,057-18,070.

Wdowinski, S., Bock, Y., Baer, G., Prawirodirdjo, L., Bechor, N., Naaman, S., Knafo, R., Forrai, Y. \& Melzer, Y. (2004) GPS measurements of current crustal movements along the Dead Sea Fault, J. Geophys. Res., 109, B05403, 10.1029/2003JB002640.

Zhang, J., Y. Bock, H. Johnson, P. Fang, S. Williams, J. Genrich, S. Wdowinski, and J. Behr (1997), Southern California Permanent GPS Geodetic Array: Error Analysis of Daily Position Estimates and Site Velocities, J. Geophys. Res., 102 (B8), $18,035-18,055$.

Zumberge, J. F., Heflin, M. B., Jefferson, D. C., \& Watkins, M. M. (1997). Precise point positioning for the efficient and robust analysis of GPS data from large networks, J. Geophys. Res., 102, 5,005-5,017. 
Table 1

GPS softwares, CGPS processing strategies, products (orbits, clocks and Earth rotation parameters) and receiver antenna phase centre (APC) models used by the different ESEAS CGPS Analysis Centres (ACs). Reduced version of Table 2 in Kierulf et al. (2006).

\begin{tabular}{cllll} 
AC & \multicolumn{1}{c}{ Software } & Strategy & Products & APC \\
\hline GCM & GAMIT/GLOBK $^{\mathrm{a}}$ & RN-DD $^{\mathrm{b}}$ & IGS $^{\mathrm{c}}$ & relative \\
NMA & GIPSY OASIS II $^{\mathrm{d}}$ & PPP $^{\mathrm{e}}$ & JPL $^{\mathrm{f}}$ & absolute \\
ROA & GIPSY OASIS II & PPP & JPL & relative \\
SRC & Bernese 4.2 & RN-DD & IGS & relative \\
UNOTT & Bernese 4.2 & PPP & IGS & relative \\
UPC & GIPSY OASIS II & PPP & JPL & relative
\end{tabular}

${ }^{a}$ King \& Bock (2003); Herring (2003)

${ }^{b}$ RN-DD: Regional network double differencing

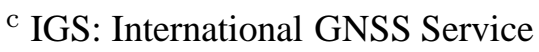

${ }^{d}$ Zumberge et al. (1997)

e PPP: Precise point positioning

f JPL: Jet Propulsion Laboratory

${ }^{g}$ Hugentobler et al. (2001) 
Table 2

Analysis-centre-, station- and CGPS processing strategy-specific mean noise amplitudes $a_{m}$ and $b_{\kappa m}$ from MLE (WN+PLN) for the period from 2000 to 2003. S. D. denotes the standard deviation. Station- and strategy-specific mean noise amplitudes have been computed excluding results from SRC and UNOTT.

\begin{tabular}{lcccc}
\hline & $a_{m}[\mathrm{~mm}]$ & S. D. & $b_{\kappa m}\left[\mathrm{~mm} \mathrm{yr}^{\kappa / 4}\right]$ & S. D. \\
\hline \multicolumn{5}{c}{ analysis-centre-specific } \\
GCM & 4.3 & 1.0 & 11.5 & 3.6 \\
NMA & 4.0 & 2.2 & 11.6 & 2.7 \\
ROA & 4.2 & 1.2 & 10.9 & 1.6 \\
SRC & 3.8 & 1.5 & 16.0 & 6.0 \\
UNOTT_flt & 3.1 & 2.0 & 15.2 & 5.1 \\
UNOTT_unflt & 4.2 & 1.8 & 20.4 & 7.7 \\
UPC & 5.3 & 1.1 & 12.8 & 2.6
\end{tabular}

station-specific

(excluding SRC and UNOTT)

$\begin{array}{lllll}\text { ALAC } & 4.6 & 0.2 & 10.0 & 0.4 \\ \text { CASC } & 4.8 & 0.6 & 11.3 & 3.9 \\ \text { GENO } & 5.6 & 1.7 & 13.0 & 4.1 \\ \text { HELG } & 0.0 & 0.1 & 12.8 & 1.9 \\ \text { LOWE } & 3.4 & 2.3 & 10.2 & 1.1 \\ \text { NEWL } & 1.4 & 1.6 & 13.1 & 1.7\end{array}$

strategy-specific

(excluding SRC and UNOTT)

\begin{tabular}{lllll} 
PPP & 4.4 & 1.6 & 11.8 & 0.8 \\
RN-DD & 4.3 & 1.0 & 11.5 & 3.6 \\
\hline
\end{tabular}


Table 3

Accumulated loadings for the results for five different ESEAS CGPS analysis centres for the period from 2000 to 2003 (in \%).

\begin{tabular}{lrrrrr}
\hline AC & GCM & NMA & ROA & SRC & UNOTT_flt \\
\hline Loading 1 & 38 & 45 & 44 & 45 & 28 \\
Loading 2 & 57 & 56 & 58 & 64 & 49 \\
Loading 3 & 70 & 66 & 67 & 75 & 61 \\
Loading 4 & 79 & 73 & 74 & 81 & 70 \\
Loading 5 & 86 & 79 & 80 & 87 & 78 \\
Loading 6 & 91 & 85 & 86 & 91 & 85 \\
Loading 7 & 95 & 91 & 92 & 95 & 91 \\
Loading 8 & 98 & 96 & 96 & 98 & 96 \\
Loading 9 & 100 & 100 & 100 & 100 & 100 \\
\hline
\end{tabular}




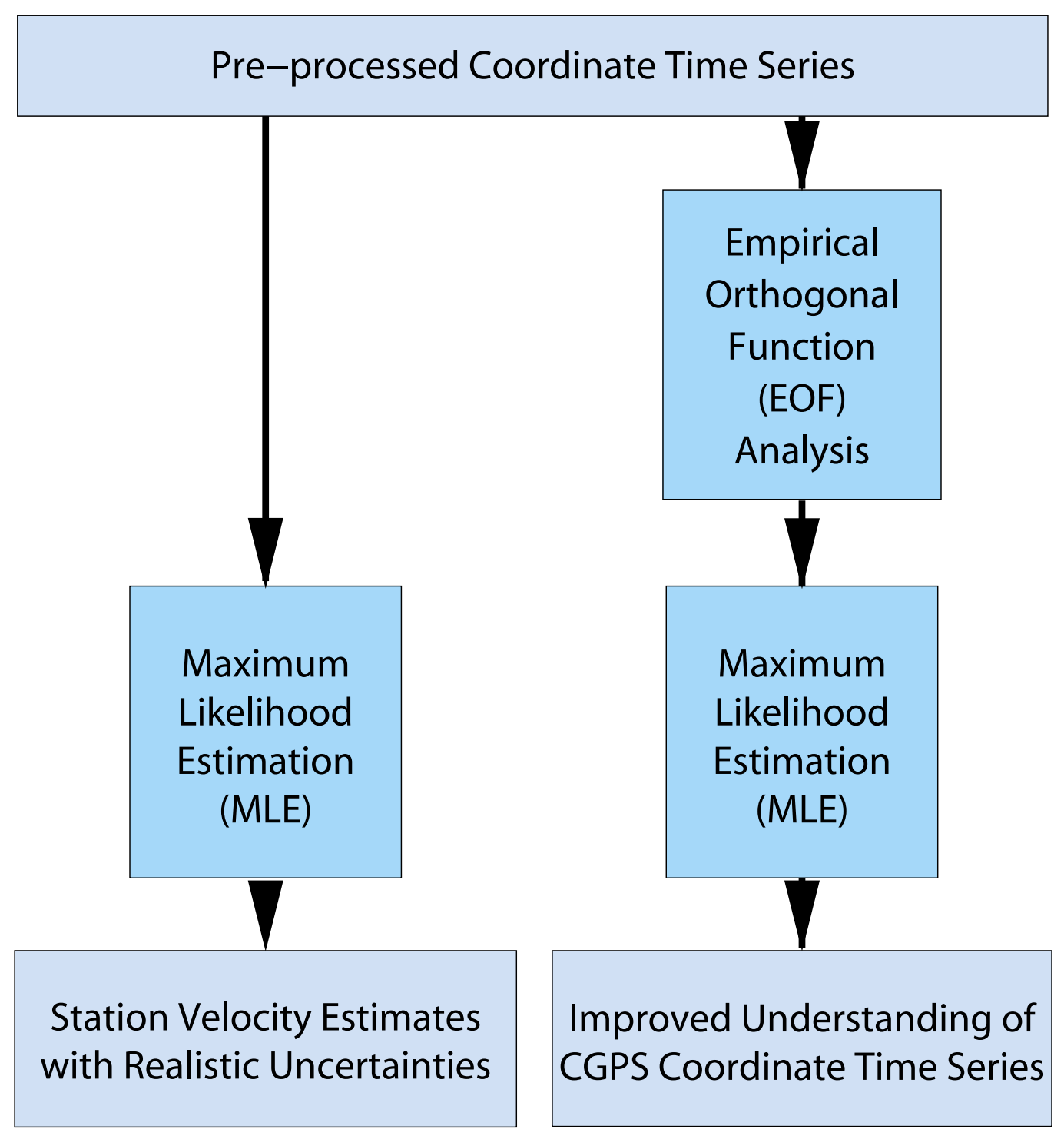

Fig. 1. Schematic of the CGPS coordinate time series analysis strategy. 


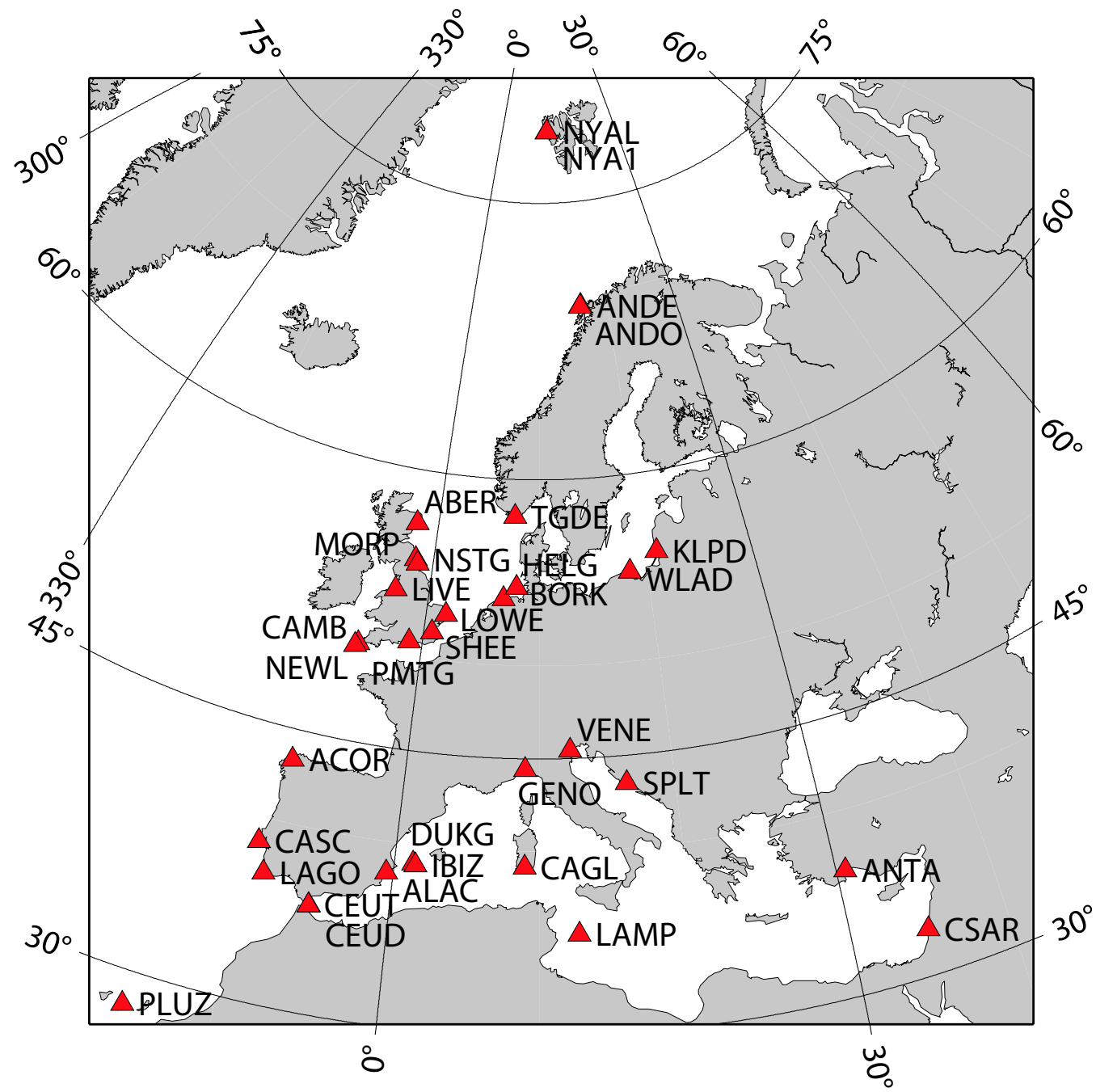

Fig. 2. Network of CGPS stations processed by the ESEAS CGPS Analysis Centres for the period 2000 to 2003. ASC1 is not shown on map. Additional stations in Malta and Greece will come on-line in 2005. 

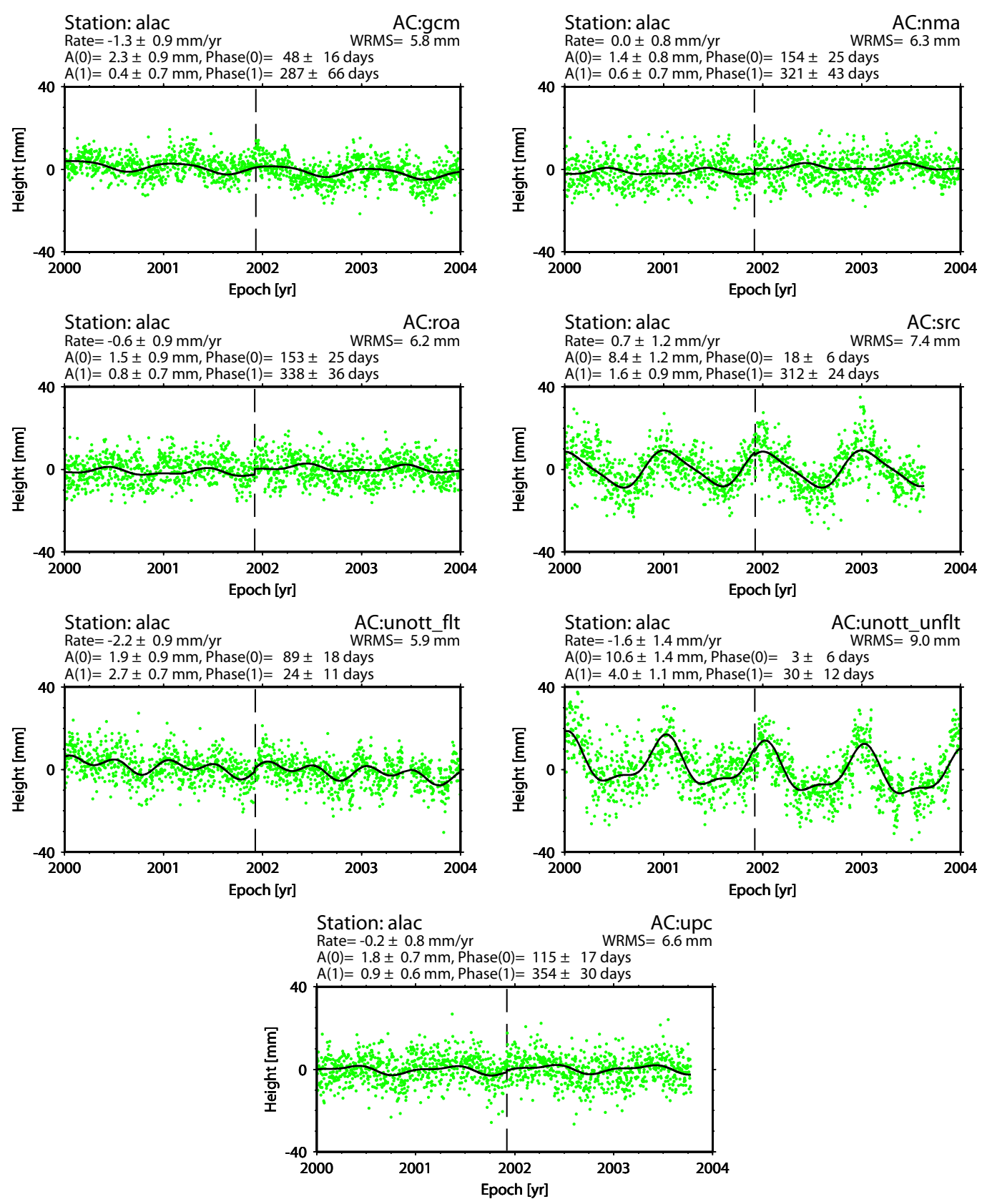

Fig. 3. Preliminary height time series for ALAC from different ESEAS CGPS analysis centres for the period 2000 to 2003 . The model parameterization of the MLE (WN+PLN) comprises a constant, a linear rate, height offsets and annual and semi-annual signals. $\mathrm{A}(0)$ and Phase(0), and A(1) and Phase(1) denote the amplitude and phase shift of the annual and semi-annual signals, respectively. The vertical dashed line depicts the modeled offset at epoch 2 December 2001. 


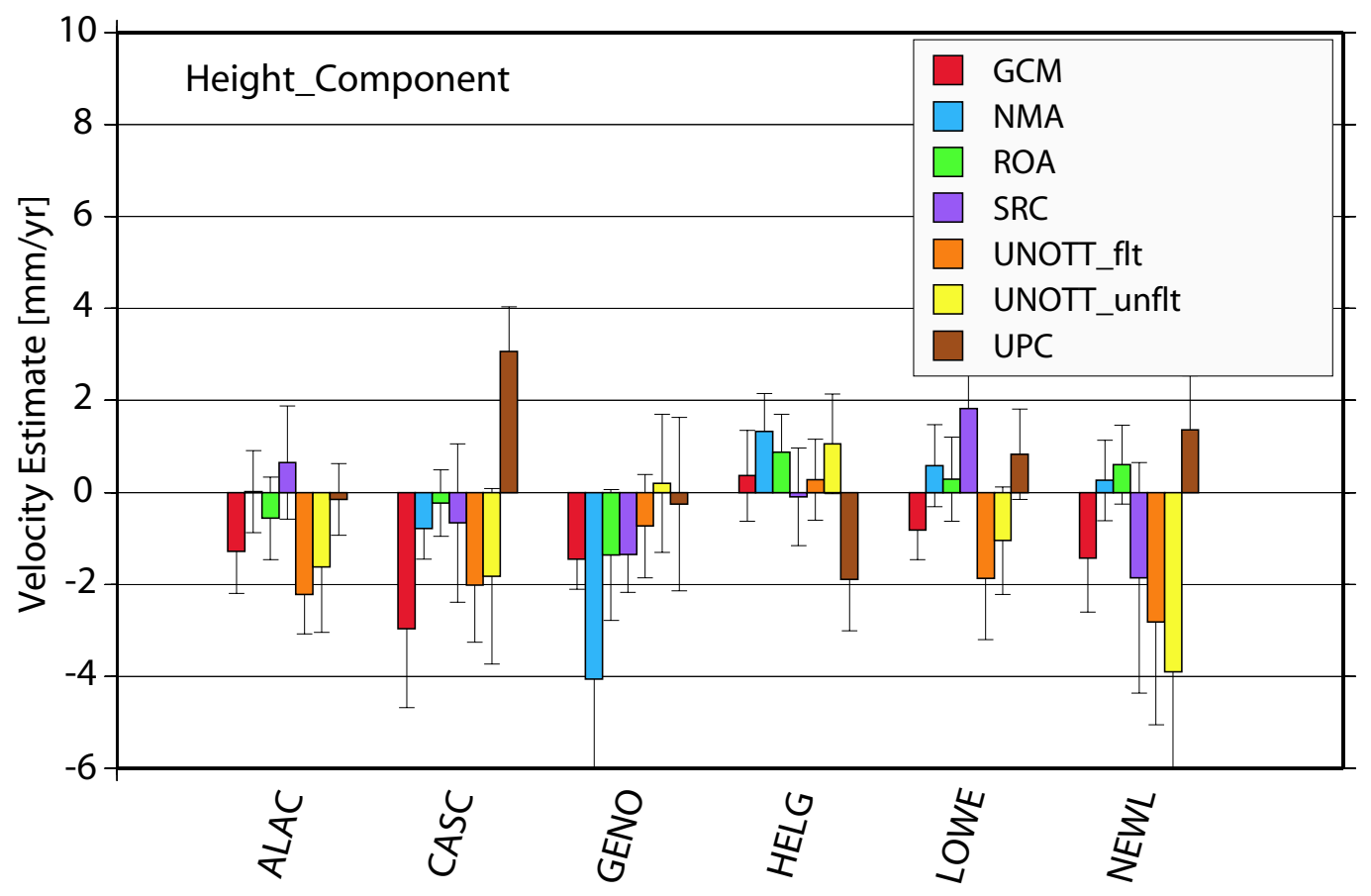

Fig. 4. Preliminary vertical station velocity estimates from MLE (WN+PLN) for different ESEAS CGPS analysis centres for the period 2000 to 2003. The uncertainties are 1- $\sigma$. 


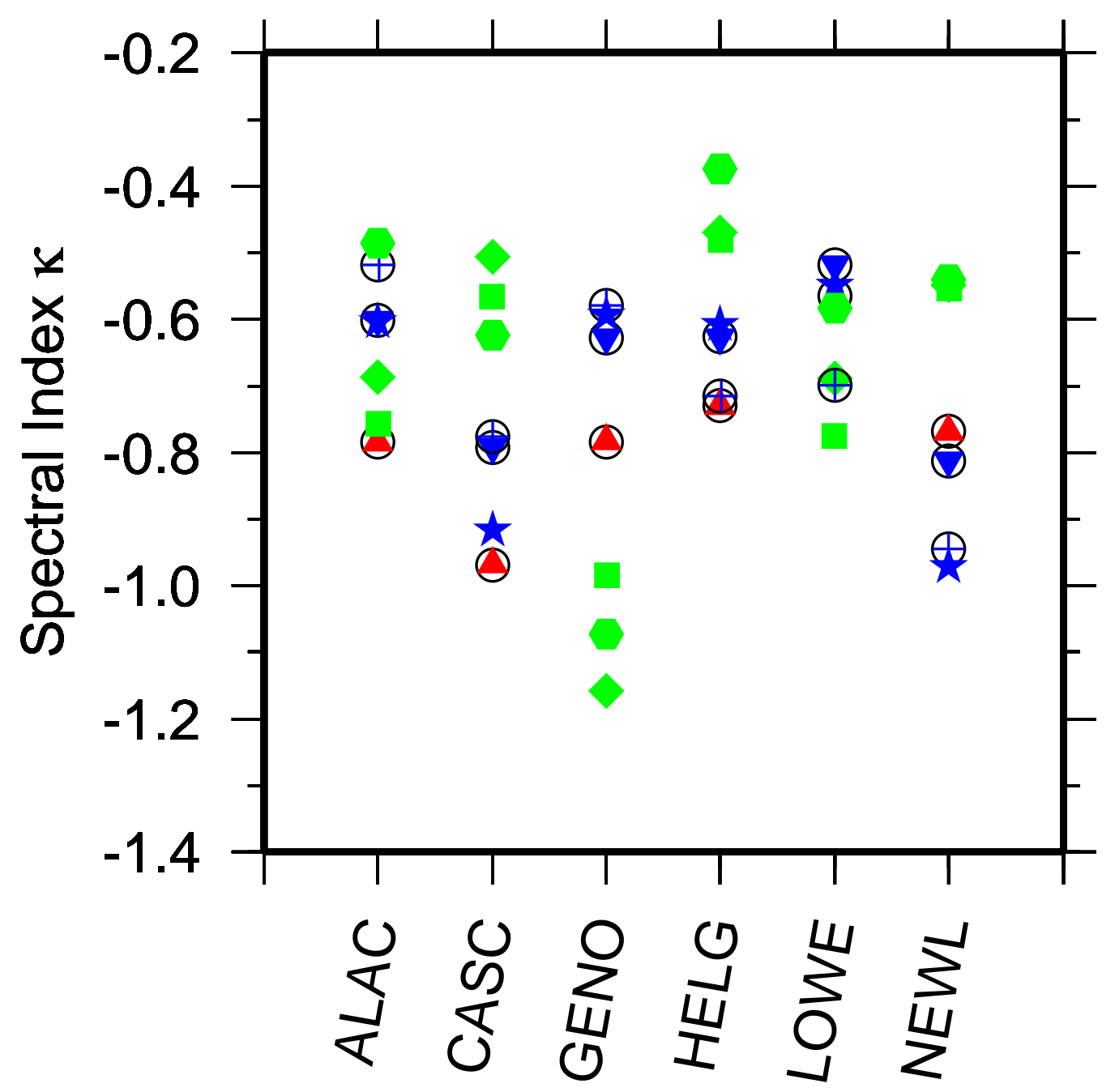

Fig. 5. Spectral indices $\kappa$ from MLE (WN+PLN) for the height time series for six CGPS stations for the period from 2000 to 2003. Symbols identify the ESEAS CGPS analysis centres (GCM: triangle, NMA: diamond, ROA: square, SRC: inverted triangle, UNOTT flt: cross, UNOTT_unflt: star, UPC: hexagon). Colour identifies different GPS softwares (Bernese 4.2: blue, GAMIT: red, GIPSY OASIS II: green). Circled symbols identify RN-DD or regionally filtered solutions. 

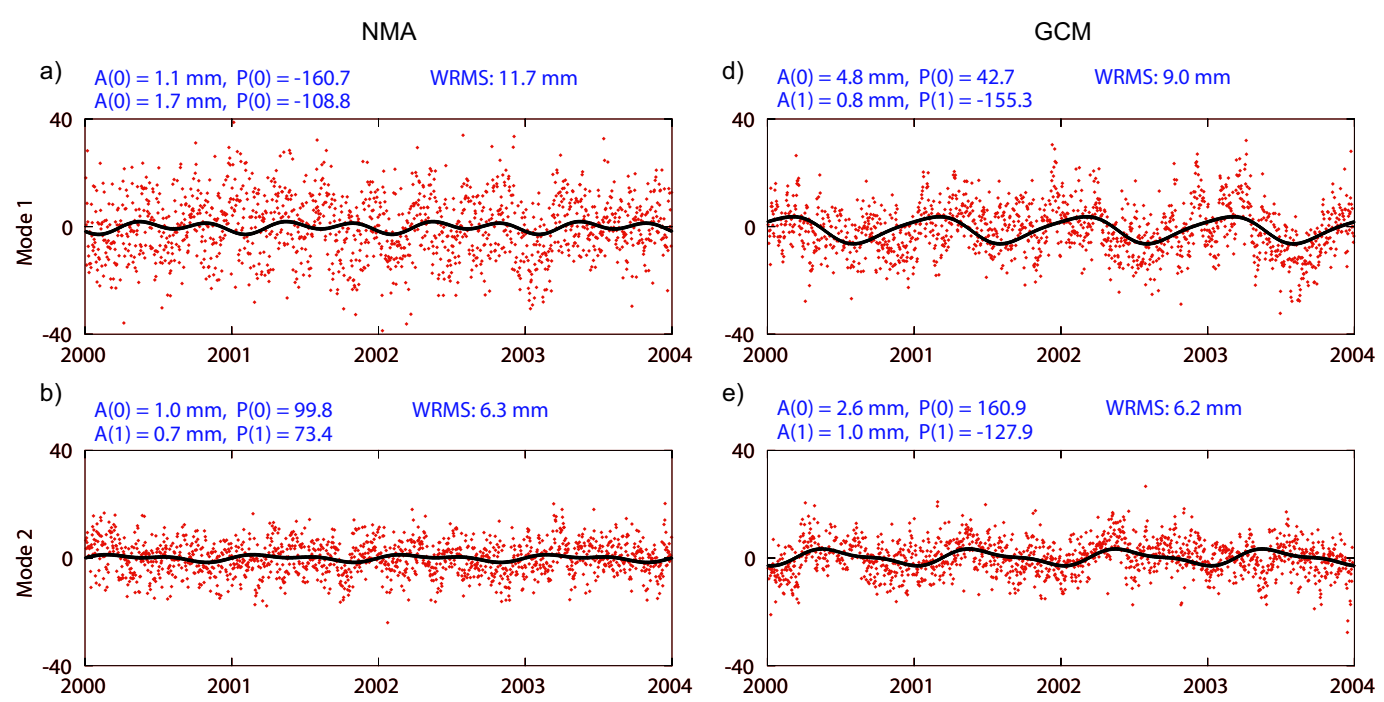

e) $\mathrm{A}(0)=2.6 \mathrm{~mm}, \mathrm{P}(0)=160.9 \quad$ WRMS: $6.2 \mathrm{~mm}$
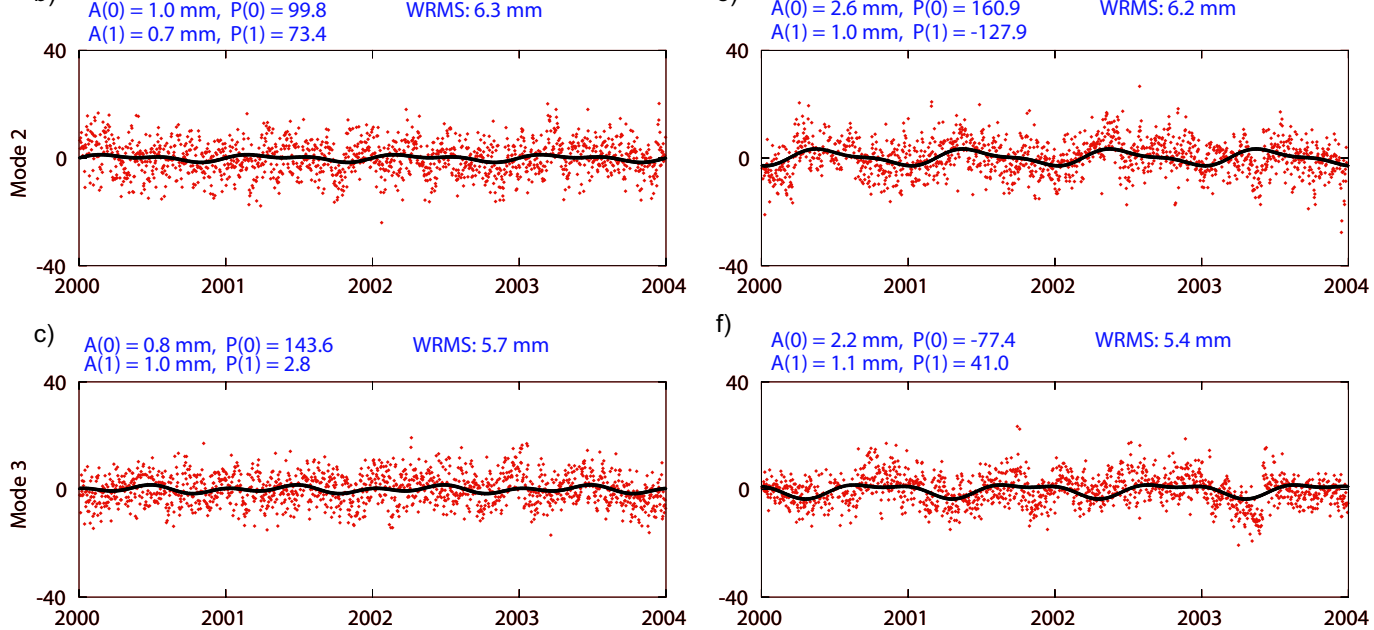

Fig. 6. Time series of the 1st, 2nd and 3rd mode based on the preliminary height time series, for the period from 2000 to 2003, for nine CGPS stations from ESEAS CGPS analysis centres NMA (left column) and GCM (right column). NMA and GCM apply PPP and RN-DD solutions, respectively (Tab. 1). The parameterization of the least-squares model fit to the common mode time series comprises a constant, a linear rate and annual and semi-annual signals. $\mathrm{A}(0)$ and $\mathrm{P}(0)$, and $\mathrm{A}(1)$ and $\mathrm{P}(1)$ denote the amplitude and phase shift of the annual and semi-annual signals, respectively. 

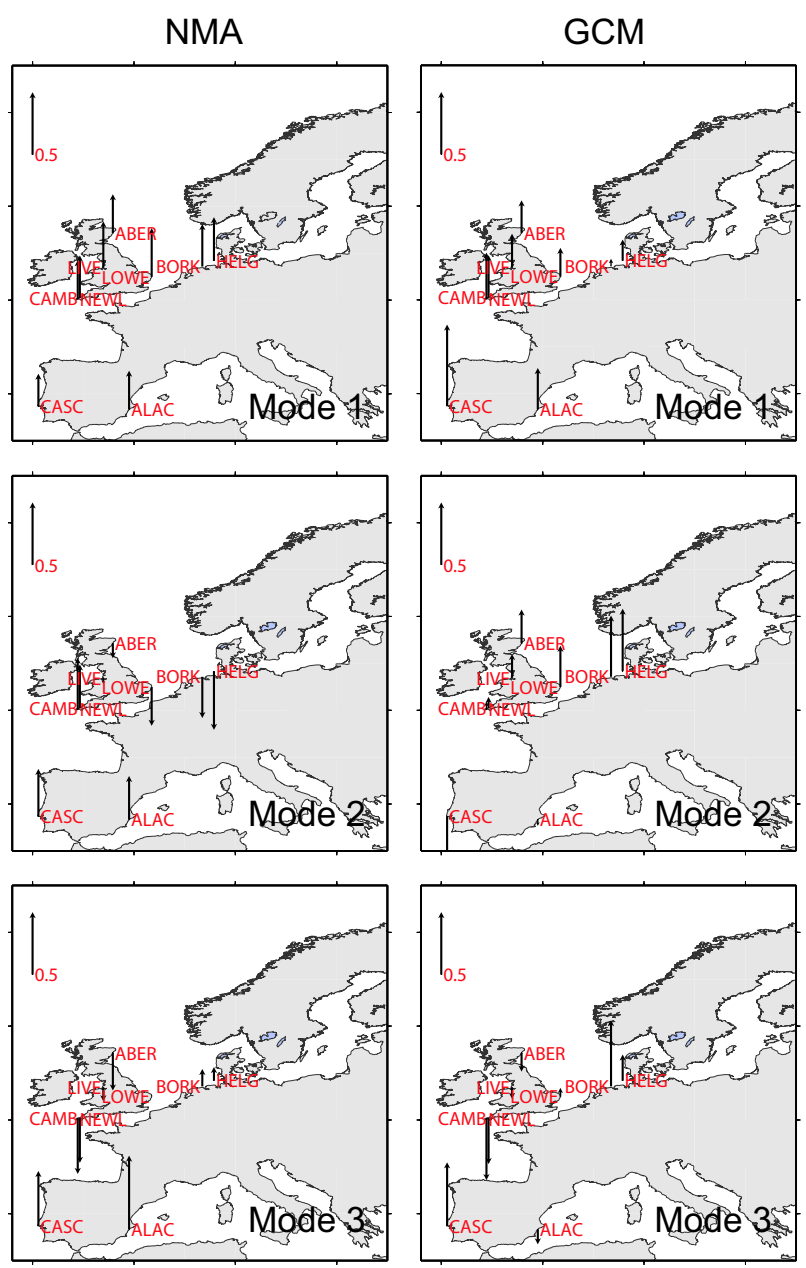

Fig. 7. Spatial pattern of the 1st, 2nd and 3rd mode based on the preliminary height time series. for the period from 2000 to 2003, for nine CGPS stations from ESEAS CGPS analysis centres NMA (left column) and GCM (right column). NMA and GCM use PPP and RN-DD solutions, respectively (Tab. 1). 